\title{
Frecuencia y factores de riesgo de la culminación de partos por cesárea en un hospital privado de la Ciudad de México
}

\author{
Frequency and risk factors for cesarean delivery \\ completion in a private hospital in Mexico City
}

\author{
Martín Salinas Pérez, * Samuel Santoyo Haro, ${ }^{\ddagger}$ \\ Ignacio Lara Barragán Bernal§
}

\begin{abstract}
Citar como: Salinas PM, Santoyo HS, Lara BBI. Frecuencia y factores de riesgo de la culminación de partos por cesárea en un hospital privado de la Ciudad de México. Acta Med GA. 2021; 19 (4): 510-513. https://dx.doi.org/10.35366/102537
\end{abstract}

\section{Resumen}

La cesárea es un procedimiento vital cuando el parto vaginal representa un riesgo para la madre o el bebé. La Organización Mundial de la Salud establece que la tasa ideal de cesárea se sitúa entre 10 y 15\%. La caracterización de este fenómeno resulta importante para plantear estrategias preventivas realistas, pues su aplicación desmedida es un problema de salud pública mundial. Estudio transversal, observacional y retrospectivo. Se analizó la información de todos los eventos obstétricos atendidos de marzo de 2019 a agosto de 2020, analizando frecuencias y factores de riesgo. Se reportaron 714 eventos obstétricos en 17 meses. La tasa de cesáreas fue de 83\%. Las diferencias entre las mujeres intervenidas por cesárea y las que culminaron su embarazo por vía vaginal, fueron la edad materna, las semanas de gestación y el número de gestas. La tasa de cesáreas en el sector privado está por arriba de las recomendaciones de la OMS. Puede pensarse que es factible una reducción de esta tendencia, dado que los partos vaginales fueron mayores en mujeres más jóvenes y primigestas, apelando a la propensión de las nuevas generaciones a respetar los medios naturales, lo que el obstetra puede capitalizar para salvaguardar el bienestar del binomio materno/fetal.

Palabras clave: Cesárea, factores de riesgo, servicios de salud, muerte materna.

\section{Abstract}

Cesarean section (C-section) is a vital procedure when vaginal delivery poses a risk to the mother or the baby. The World Health Organization (WHO) states that the ideal C-section rate is between 10 and $15 \%$. The characterization of this phenomenon is important for developing realistic preventive strategies, as their unmeasured realization is a global public health problem. Cross-sectional, observational, and retrospective study. Information on all obstetric events attended from March 2019 to August 2020 was analyzed, examining frequencies and risk factors. 714 obstetric events were reported in 17 months. The $\mathrm{C}$-section rate was $83 \%$. The differences between $\mathrm{C}$-section-intervened women and those who completed their pregnancy vaginally were maternal age, weeks, and number of gestations. The rate of $\mathrm{C}$-sections in the private sector is above $\mathrm{WHO}$ recommendations. A reduction of this trend can be thought feasible, since vaginal deliveries were higher in younger and primiparous women, appealing to the trend in new generations to respect the natural environment, which can be capitalized by the obstetrician to safeguard the welfare of the mother/fetal binomial.

Keywords: Caesarean sections, risk factors, health services, maternal death.
* Alumno de la Facultad Mexicana de Medicina de la Universidad La Salle, México.

₹ Profesor titular del curso de Ginecología y Obstetricia, Hospital Angeles Pedregal. México.

$\S$ Profesor adjunto del curso de Ginecología y Obstetricia, Hospital Angeles Pedregal. México.
Correspondencia:

Martín Salinas Pérez

Correo electrónico: salinas.martin@hotmail.com

Aceptado: 12-03-2021.

www.medigraphic.com/actamedica 


\section{INTRODUCCIÓN}

En 1985 la Organización Mundial de la Salud (OMS) propuso que del total de los nacimientos, el porcentaje de cesáreas debería ser de entre 10 y 15\%; un porcentaje inferior a 5\% sugeriría una limitación en la realización de cesáreas, mientras que un porcentaje mayor de cesáreas al señalado no representaría beneficios adicionales. ${ }^{1}$ Cuando la cesárea está justificada desde el punto de vista médico, es eficaz para prevenir la morbimortalidad materna y perinatal. Sin embargo, no están demostrados los beneficios del parto por cesárea para las mujeres o los neonatos en quienes este procedimiento resulta innecesario. Como en cualquier otra cirugía, la cesárea está asociada con riesgos a corto y a largo plazo que pueden perdurar por muchos años después de la intervención y afectar la salud de la mujer y del neonato así como a cualquier embarazo futuro. ${ }^{2,3}$ La proporción de cesáreas a nivel de población es una medida del nivel de acceso a esta intervención y de su uso. Puede funcionar como guía para que los gobiernos y los responsables de la formulación de políticas evalúen los avances en la salud maternoinfantil y controlen la atención obstétrica de emergencia y la utilización de los recursos. ${ }^{4}$ A comienzos de la década de los 90, las tasas elevadas de cesáreas eran un fenómeno propio de los países latinoamericanos. En México las tasas de cesáreas realizadas fluctuaban entre $38 \%$ en la población no derechohabiente y $50 \%$ en la población con algún tipo de seguridad social. Hoy en día, esta "epidemia" se ha extendido al mundo entero. ${ }^{5}$ En México, las principales causas de muerte materna son los trastornos hipertensivos asociados al embarazo, parto y puerperio, hemorragia, trastornos tromboembólicos y sepsis. ${ }^{6,7}$ Controversialmente, las evidencias sustentadas con rigor metodológico establecen que el nacimiento por cesárea está asociado con complicaciones, entre las más frecuentes se encuentran las mismas causas de muerte señaladas, excepto por los trastornos hipertensivos, es decir: infecciones, hemorragia, fenómenos tromboembólicos y se agregan lesiones viscerales. Las complicaciones oscilan entre 12 y $15 \% .{ }^{8}$ Diversos estudios en diferentes países han señalado que los factores de incremento del porcentaje de las cesáreas son multifactoriales y difíciles de estudiar. ${ }^{9}$ Entre los factores identificados se señalan, en primer lugar, los cambios en las características maternas y su relación con la práctica obstétrica que se ha modificado debido a las preocupaciones relacionadas con el feto y la seguridad de la madre, ${ }^{10}$ seguidos de los factores sociales e institucionales. ${ }^{11}$ El objetivo de este estudio fue identificar la frecuencia así como los factores de riesgo de la culminación de partos por cesárea en un hospital privado de la Ciudad de México.

\section{MATERIAL Y MÉTODOS}

Se realizó un estudio observacional, transversal y retrospectivo, incluyendo todos los expedientes de mujeres que acudieron al Hospital Angeles Pedregal en un periodo de marzo de 2019 a agosto de 2020 para ser atendidas por un evento obstétrico. Se identificaron los expedientes de las mujeres a las que se les practicó un parto por cesárea y se realizó un análisis de causas. Se compararon las características demográficas entre las mujeres intervenidas con cesárea y aquéllas que culminaron su embarazo vía vaginal. Se elaboró una base de datos en el programa estadístico SPSS. Se utilizaron medidas de frecuencia para presentar las variables cualitativas. Se realizaron análisis de normalidad para las variables cuantitativas y se utilizaron medidas de tendencia central y dispersión para su presentación en tablas. El contraste, se realizó la prueba t de Student para la diferencia entre medias, $U$ de Mann-Whitney para la diferencia entre medianas y la prueba $\chi^{2}$ para determinar la diferencia entre porcentajes.

\section{RESULTADOS}

Se incorporó un total de 714 expedientes correspondientes a los eventos obstétricos atendidos de marzo de 2019 a agosto de 2020. El total de cesáreas realizadas fue de 597, lo que corresponde a una tasa de $84 \%$ sobre el total de nacimientos versus $16 \%$ de partos atendidos por vía vaginal. La mediana de la edad en las mujeres atendidas por parto vaginal fue menor que la de las mujeres atendidas por cesárea $(p=0.003)$. La edad gestacional fue menor en el grupo de cesárea. En cuanto al número de gestas, se observó que $51.3 \%$ de las atendidas por parto vaginal fueron primigestas, en cambio en el grupo de cesáreas sólo $38.5 \%$ fueron primigestas y el resto (la mayoría) fueron secundigestas, tres gestas o más $61.5 \%$ (Tabla 1).

En cuanto a las causas más frecuentes se reportó en primer lugar el diagnóstico de cesárea previa (14.4\%), seguido de cesárea electiva (9.1\%) y desproporción cefalopélvica (7.6\%) (Tabla 2) .

\section{DISCUSIÓN Y CONCLUSIONES}

Según Heffner, de las condiciones maternas que determinan la vía de nacimiento, destacan la edad materna y la reducción en el número de hijos deseados. ${ }^{12}$ En congruencia, nuestros hallazgos apuntan a que a mayor edad mayor es la proporción de cesáreas realizadas, probablemente aunado al hecho que involucra una paridad satisfecha, que en el caso de nuestro estudio resultó ser la causa de $5 \%$ de las cesáreas realizadas. Asimismo, coincidimos con otros autores al evidenciar que la edad 
Tabla 1: Características de las madres que tuvieron un evento obstétrico en el periodo de marzo de 2019-agosto de 2020. $(\mathrm{N}=714)$.

\begin{tabular}{|c|c|c|c|}
\hline & Cesárea $(\mathrm{N}=597)$ & Parto vaginal $(\mathrm{N}=117)$ & $\mathrm{p}$ \\
\hline Edad materna (años)* & $34(31-37)$ & $33(30-35)$ & 0.003 \\
\hline Edad gestacional (semanas) ${ }^{\star \star}$ & $37.7 \pm 1.6$ & $38.2 \pm 1.6$ & 0.004 \\
\hline Primigesta & $38.50 \%$ & $51.30 \%$ & 0.035 \\
\hline Secundigesta & $34.60 \%$ & $36.80 \%$ & \\
\hline Tres gestas o más & $27.00 \%$ & $12.00 \%$ & \\
\hline
\end{tabular}

\begin{tabular}{lr}
\hline \multicolumn{2}{c}{$\begin{array}{c}\text { Tabla 2: Principales diagnósticos para la } \\
\text { indicación de parto por cesárea. (N = 597). }\end{array}$} \\
& $\%$ \\
\hline & \\
\hline Cesárea previa & 14.4 \\
Cesárea electiva & 9.1 \\
Desproporción cefalopélvica & 7.6 \\
Falta de progresión en el TP & 7.3 \\
Cesárea previa + EMR + paridad satisfecha & 5.1 \\
Pélvico & 4.9 \\
Enfermedad hipertensiva del embarazo & 4.9 \\
Baja reserva fetal & 4.9 \\
Iterativa & 4.9 \\
Otros & 36.9 \\
\hline TP = trabajo de parto; EMR= edad materna de riesgo. & \\
\hline
\end{tabular}

gestacional menor de 38 semanas de gestación o mayor de 40 semanas aumenta la probabilidad de tener un nacimiento por cesárea.

En la década de los 50 Redd D trabajó sobre miedo y tensión al dolor. Argumentó en sus estudios que el miedo es causado por experiencias previas y las expectativas de que el parto siempre resulta doloroso. ${ }^{13}$ Estos aspectos (específicamente el haber vivido una experiencia previa traumática) determinaron las corrientes iniciales de la psicoprofilaxis y pueden ser la explicación de que en nuestra población de estudio 53\% de las mujeres que tuvieron un parto vaginal fueron primigestas; sin embargo, más de $60 \%$ de las mujeres que se intervinieron por cesárea habían tenido dos gestas o más.

Según datos presentados por Uribe y colaboradores en 2018, ${ }^{14}$ la tasa de parto por cesárea en México estaba entre las más altas del mundo, ésta aumentó de $43.9 \%$ $(869,018 / 1,978,380)$ en 2016 a $45.5 \%$ (940,206/2,064,507) en 2018, lo que representó un aumento porcentual relativo de la tasa de $3.7 \%$. El mayor cambio en las tasas de partos se produjo en los hospitales del sector privado. La tasa obtenida en nuestro estudio rebasa las estimaciones nacionales, observándose este fenómeno en $84 \%$ sobre el total de nacimientos.

Actualmente, el sistema de salud cubano identifica y refleja como problemas actuales que podrían determinar la calidad de la atención al parto: insuficiente capacitación del personal de salud en aspectos bioéticos y de humanización, desactualización de temas sobre la conducta, el control del trabajo de parto relacionado con el dolor, estrés, tensión de la madre, desarrollo casi nulo de la psicoprofilaxis, problemas estructurales, dificultades organizativas, obstáculos en la calidad de la información general y sobre procedimientos y conductas con escaso empleo del consentimiento informado en la práctica cotidiana. ${ }^{15,16}$

La OMS en su documento Recomendaciones para la apropiada utilización de la tecnología en el nacimiento, Declaración de Fortaleza redactada en 1985, subraya que "no existe justificación para una tasa de cesárea superior a 10-15\%" y que "se debe favorecer el nacimiento por vía vaginal en pacientes con cesárea previa". Las guías de práctica clínica (GPC) son un instrumento apropiado para reducir la frecuencia de la operación cesárea. Su constante actualización mediante la unificación de criterios en las indicaciones de la cirugía, así como su aplicación en una población específica, contribuye a disminuir su práctica injustificada. ${ }^{1}$

A criterio de los autores, una de las propuestas que podrían generarse a partir de los datos obtenidos en este estudio (primiparidad y edad menor de las pacientes que tuvieron un parto vaginal) es la de incorporar a los ser- 
vicios de atención del parto la recomendación de parto humanizado, mismo que obedece a un proceso histórico mundial como una respuesta frente al modelo convencional medicalizado que desestima el proceso natural de las mujeres y no toma en cuenta sus necesidades emocionales, culturales y sociales, la perspectiva de género, ni las recomendaciones sustentadas en el enfoque basado en evidencias y los principios propuestos por la OMS. Este modelo de parto humanizado ha mostrado excelentes resultados en países como Argentina. La adquisición de salas labor/ parto/recuperación (LPR) podría en cierta medida brindar una experiencia distinta a aquellas mujeres que en algún momento pudieran haber optado por una cesárea electiva, esta opción permite que el periodo de labor o trabajo de parto, el nacimiento del bebé y la recuperación de la madre después del parto se hagan en el mismo lugar sin necesidad de mover a la mujer de un lado para otro.

Sin lugar a dudas las ventajas de un parto vaginal sobre una operación cesárea se han demostrado históricamente; sin embargo, no hemos logrado que este concepto de bienestar materno/fetal sea comprendido por la comunidad, que ha priorizado la comodidad materna al percibir a esta última como un procedimiento rápido e inocuo. Así pues, el proyecto de acción relacionado con los problemas de humanización de la atención al parto no puede circunscribirse sólo a la actualización de los conocimientos clínicos y tecnológicos, sino que debe incluir diferentes formas de transformar el conocimiento teórico que sustente en la práctica la expresión de los conocimientos de una forma integradora, donde el humanismo avance de forma paralela a los adelantos científicos y tecnológicos.

\section{REFERENCIAS}

1. Appropriate technology for birth. Lancet. 1985; 2(8452): 436-437.

2. Organización Mundial de la Salud. Declaración de la OMS sobre las tasas de cesáreas. Ginebra, Suiza: OMS; 2015. [Acceso 28 de diciembre de 2020] Disponible en: https://www.who.int/ reproductivehealth/publications/maternal_perinatal_health/cs-statement/ es/\#: : :text=Desde\%201985\%2C\%20los\%20profesionales\%20 de,como\%20en\%20pa\%C3\%ADses\%20en\%20desarrollo
3. Ye J, Betrán AP, Guerrero Vela M, Souza JP, Zhang J. Searching for the optimal rate of medically necessary cesarean delivery. Birth. 2014; 41(3): 237-244.

4. World Health Organization, UNFPA, UNICEF, Mailman School of Public Health. Monitoring emergency obstetric care: a handbook. Geneva, Switzerland: World Health Organization; 2009.

5. Ecker JL, Frigoletto FD Jr. Cesarean delivery and the risk-benefit calculus. N Engl J Med. 2007; 356(9): 885-888.

6. Angulo VJ, Cortés SL, Torres GLG, Aguayo AG, Hernández HS, Ávalos NJ. Análisis de la mortalidad materna, en tres periodos, en el Hospital de Ginecología y Obstetricia del Centro Médico Nacional de Occidente. Ginecol Obstet Mex. 2007; 75(7): 384-393.

7. García-Regalado JF, Montañez-Vargas MI. Signos y síntomas de alarma obstétrica Acerca del conocimiento que tienen las mujeres. Rev Med Inst Mex Seguro Soc. 2012; 50(6): 651-657.

8. Karchmer KS, Fernández-del Castillo C. Obstetricia y medicina perinatal. Temas selectos. México: Colegio Mexicano de Especialistas en Ginecología y Obstetricia; 2006.

9. Gunnervik C, Sydsjo G, Sydsjo A, Selling KE, Josefsson A. Attitudes towards cesarean section in a nationwide sample of obstetricians and gynecologists. Acta Obstet Gynecol Scand. 2008; 87(4): 438-444.

10. Joseph KS, Young DC, Dodds L, O'Connell CM, Allen VM, Chandra $\mathrm{S}$ et al. Changes in maternal characteristics and obstetric practice and recent increases in primary cesarean delivery. Obstet Gynecol. 2003; 102(4): 791-800.

11. Kabir AA, Steinmann WC, Myers L, Khan MM, Herrera EA, Yu S et al. Unnecessary cesarean delivery in Louisiana: an analysis of birth certificate data. Am J Obstet Gynecol. 2004; 190(1): 10-19.

12. Heffner LJ, Elkin E, Fretts RC. Impact of labor induction, gestational age, and maternal age on cesarean delivery rates. Obstet Gynecol. 2003; 102(2): 287-293.

13. Borges Damas, Sánchez Machado R, Domínguez Hernández R, Sixto Pérez A. El parto humanizado como necesidad para la atención integral a la mujer. Rev Cubana Obstet Ginecol. 2018; 44(3): 1-12.

14. Uribe-Leitz T, Barrero-Castillero A, Cervantes-Trejo A, Santos JM, de la Rosa-Rabago A, Lipsitz SR et al. Trends of caesarean delivery from 2008 to 2017, Mexico. Bull World Health Organ. 2019; 97(7): 502-512.

15. Ruiz-Sánchez J, Espino y Sosa S, Vallejos-Parés A, Durán-Arenas L. Cesárea: tendencias y resultados. Perinatol Reprod Hum. 2014; 28(1): 33-40.

16. Águila Setién S, Breto García A, Cabezas Cruz E, Delgado Calzado JJ, Santisteban Alba E. Obstetricia y perinatología diagnóstico y tratamiento. 2a ed. Cuba: Editorial Ciencias Médicas; 2014. pp. 66-68.

17. Instituto Mexicano del Seguro Social. Guía de Práctica Clínica para la Reducción de la Frecuencia de Operación Cesárea. México: Instituto Mexicano de Seguro social; 2009, actualización 2014. Disponible en: http://www.imss.gob.mx/sites/all/statics/guiasclinicas/048GER.pdf 